\title{
Gut microbiota in Mexican patients with common variable immunodeficiency
}

\author{
Ana Paola Franco-Esquivias, ${ }^{1}$ Cristina García-De la Peña, ${ }^{2 *}$ Carlos Torres-Lozano, ${ }^{1}$
} Felipe Vaca-Paniagua, ${ }^{3}$ Clara Díaz-Velásquez, ${ }^{3}$ Margarita Ortega-Cisneros ${ }^{1}$, and Antonio Quintero-Ramo ${ }^{4}$ ${ }^{1}$ Instituto Mexicano del Seguro Social, Centro Médico Nacional de Occidente, Specialty Hospital, Department of Clinical Allergy and Immunölogy, Guadalajara, Jalisco; '2Universidad Juárez del Estado de Durango, Faculty of Biological Sciences, Laboratory of Preservation Medicine, Gömez Palacio, Durango; ${ }^{3}$ Universidad Nacional Autónoma de México, Iztacala Higher Education Faculty, National Health Laboratory: Molecular Diagnosis and Environmental Effect on Chronic-Degenerative Diseases, Tlalnepantla, Estado de México; ${ }^{4}$ Universidad de Guadalajara, Health Sciences University Center, Physiology Department, Guadalajara, Jalisco. Mexico

\begin{abstract}
Introduction: Common variable immunodeficiency (CVID) is the main symptomatic primary immunodeficiency and is associated with complex immune disorders. Gut microbiota interacts closely with the immune system, and intestinal dysbiosis is related to multiple diseases. Objective: To describe for the first time the composition of gut microbiota in Mexican patients with CVID. Methods: Fecal samples from five patients with CVID were collected and massive sequencing of the V3-V4 region of $16 S$ rRNA gene was carried out using illumina technology. Results: Bacterial relative abundance was observed at alitaxonomic levels. Firmicutes, Actinobacteria and Verrucomicrobia were the predominant phyla. The Clostridia class an $\$$ the Clostridial order were the most common in their respective taxon; the Ruminococcaceae family predominated. A total of 166 genera were reported, with the most abundant being Faecalibacterium. Five species were identified, but only Bifidobacterium longum was present in all patients. Conclusions: Unlike healthy subjects' gut microbiota, where Firmicutes and Bacteroidetes predominate, the microbiota of the patients with CVID considered in this study was abundant in Firmicutes, Actinobacteria and Verrucomicrobia. The low presence of Bacteroidetes and high abundance of Firmicutes might indicate the existence of intestinal dysbiosis in these patients.
\end{abstract}

KEY WORDS: Gut microbiota. Common variable immunodeficiency. $16 S$ rRNA. Firmicutes. Dysbiosis.

\section{Introduction}

Common variable immunodeficiency (CVID) is the most common primary symptomatic immunodeficiency in the world. ${ }^{1}$ Its diagnosis is based on the criteria proposed by the American and European immunodeficiency societies: a decrease in immunoglobulin $G$ ( $\lg G)$ serum levels below two standard deviations for age and decrease in other serum immunoglobulin (IgA or $\lg M)$, age older than two years, absence of isohemagglutinins or poor response to vaccines and exclusion of other causes of hypogammaglobulinemia. ${ }^{1}$

The main clinical manifestation in CVID is tious; however, other complications such as autoimmunity, gastrointestinal disorders, granulomatossis, lymphoproliferation and neoplasms are common. ${ }^{-3}$ The characteristic immune alteration is hypogammaglobulinemia with B lymphocyte involvement; however, involvement includes other cells such as CD4+ T lymphocytes, regulatory lymphocytes (Treg CD25+
Correspondence:

${ }^{*}$ Cristina García De la Peña

E-mail: cristina.garcia@ujed.mx
Date of reception: 03-12-2018

Date of acceptance: 17-05-2019

DOI: 10.24875/GMM.M20000330
Gac Med Mex. 2019;155:447452

Contents available at PubMed

www.gacetamedicademexico.com license (http://creativecommons.org/licenses/by-nc-nd/4.0/). 
FoxP3+ lymphocytes), CD8 T lymphocytes, monocytes, antigen-presenting cells and natural killer (NK) cells. ${ }^{1,2,4}$ CVID has been associated with various genetic and environmental factors; however, none of them has been able to fully explain the multiple clinical manifestations, severity and evolution of the disease., ${ }^{1,2}$

Human intestinal microbiota has been proposed as a key micro-ecosystem in the health-disease process, where dysbiosis is associated with obesity, infections, autoimmunity and allergic diseases..$^{5-7}$ Gut microbiota evolution, development and diversity starts since birth and is modified throughout life. ${ }^{8}$ Ninety percent of the microorganisms that colonize the human intestine are represented by Firmicutes, Bacteroides, Actinobacteria, Fusobacteria, Protebacteria and Verrucomicrobia phyla. $^{9,10}$ This wide bacterial diversity has important functions in the development of the innate and adaptive immune system in a bi-directional feedback process. $^{7,11,12}$ Notwithstanding, the study of intestinal microbiota in primary immunodeficiencies is limited. Considering the amount of activities of microbiota in immune regulation and the various immune alterations of CVID, there is a need to delve into the knowledge of intestinal microbiology in patients with this medical condition. Therefore, the first analysis of the gut microbiota in Mexican patients with CVID is presented, where the structure of the intestinal microbial community associated with this pathology is described.

\section{Method}

During 2016, fecal samples were collected from five patients older than 18 years who had been diagnosed with CVID and who were under the care of the Department of Clinical Immunology and Allergy of the National Medical Center of the West, Mexican Institute of Social Security, in Guadalajara, Jalisco, Mexico. The protocol was approved by the ethics committee of that institution.

A stool sample per patient was collected using a sterile container; $0.25 \mathrm{~g}$ of each sample was placed in BashingBead ${ }^{\mathrm{TM}}$ lysis tubes with $750 \mu \mathrm{L}$ of Xpedition $^{\text {TM }}$ Zymo Research ${ }^{\text {TM }}$ lysate buffer/stabilizer. Each tube was placed in a cell disruptor (TerraLyzer ${ }^{\mathrm{TM}}$ ) for 30 seconds for DNA preservation.

DNA was extracted from the samples using the Soil/ Fecal DNA MiniPrep commercial kit (Zymo Research $\left.^{\mathrm{TM}}\right)$. The extracted DNA was run on $1.2 \%$ agarose gel at $80 \mathrm{~V}$ for 45 minutes in a BIO-RAD electrophoresis chamber to visualize the presence of high molecular weight DNA. Visualization was carried out in a GelMax ${ }^{\mathrm{TM}}$ photodocumenter (UVP $\left.{ }^{\circledR}\right)$. The amount of DNA per sample was measured on a Qabit ${ }^{\circledR}$ fluorometer. Amplification of the V3 and V4 regions of the $16 S$ rRNA gene was carried out, using the S-DBact-0341-b-S-17, 5'-CCTACGGGNGGCWGCAG̈-3' and SD-Bact-0785-aA-21, 5'-GACTACHVGGGTATCTAATCC-3' primers, which produce an amplicon of approximately $460 \mathrm{bp}^{13}$ These sequences were synthesized with the overhang adapters of the Illumina protocol (2017a), which produce an amplicon of approximately $550 \mathrm{bp}$.

The Illumina PCR protocol was used with $12.5 \mathrm{\mu L}$ of MyTaq ${ }^{\mathrm{TM}}$ Ready Mix $1 \mathrm{X}$ (Bioline $\left.{ }^{\circledR}\right), 1 \mu \mathrm{L}$ of each primer $(10 \mu \mathrm{M}), 5 \mu \mathrm{L}$ of DNA (50 ng total) and $5.5 \mu \mathrm{L}$ of molecular grade $\mathrm{H}_{2} \mathrm{O}$. The following cycle was used: $95{ }^{\circ} \mathrm{C}$ for three minutes; 25 cycles at $95^{\circ} \mathrm{C}$ for 30 . seconds, $55^{\circ} \mathrm{C}$ for 30 seconds, $72{ }^{\circ} \mathrm{C}$ for 30 seconds; $72{ }^{\circ} \mathrm{C}$ for five minutes in a Labnet Multigene ${ }^{\mathrm{TM}}$ Gradient PCR thermal cycler. $1 \mu \mathrm{L}$ of the PCR products was placed on a Bioanalyzer DNA 1000 chip to verify the size of the amplicon (approximately $550 \mathrm{bp}$ ). Amplicon purification was carried out with $0.8 \%$ Agentcourt $^{\circledR}$ AMPure ${ }^{\circledR}$ XP beads. Subsequently, the amplicons were labeled with Nextera XT Index Kit ${ }^{\mathrm{TM}}$ for the Greation of the libraries, using $25 \mu \mathrm{L}$ of MyTaq ${ }^{\mathrm{TM}}$ Ready Mix 1X (Bioline $\left.{ }^{\circledR}\right), 5 \mu \mathrm{L}$ of each primer (N7xx and S5xx), $5 \mu \mathrm{L}$ of DNA and $10 \mu \mathrm{L}$ of molecular grade $\vec{H}_{2} \mathrm{O}$. The following cycle was used: $95^{\circ} \mathrm{C}$ for three minutes; 10 cycles at $95{ }^{\circ} \mathrm{C}$ for 30 seconds, $55^{\circ} \mathrm{C}$ for 30. seconds, $72{ }^{\circ} \mathrm{C}$ for 30 seconds, $72{ }^{\circ} \mathrm{C}$ for five minutes. Purification of the libraries was carried out with $1.2 \%$ Agencourt $^{\circledR}$ AMPure $^{\circledR} \mathrm{XP}$ beads. $1 \mu \mathrm{L}$ of the fina library of some randomly selected PCR products was placed on a DNA 1000 Bioanalyzer chip to verify the size of the amplicon, with a size of approximately 630 bp being expected. Finally, quantification, normalization (equimolarity), library grouping and next-generation mass sequencing (MiSeq Illumina ${ }^{\circledR}$ of $2 \times 250$ paired-end readings) were performed, according to the Illumina $16 \mathrm{~S}$ protocol for metagenomics.

Sequence analysis was performed in Oracle $\mathrm{VM}$ VirtualBox 5.1.14 on the MGLinux platform using the Quantitative Insights Into Microbial Ecology bioinformatics software, version 1.9.0.14 The process was initiated by assembling the forward and reverse sequences of the samples, using the PEAR ${ }^{15}$ program with an overlap of $50 \mathrm{bp}$, a minimum length of $430 \mathrm{bp}$ per reading and maximum length of $470 \mathrm{bp}$, a quality criterion Q30 (one erroneous base per 1000 basses) and a $p$-value $<0.0001$. The files were next converted into the FASTA format and elimination of chimeric 
Table 1. Information on the bacterial sequences used in the present study

\begin{tabular}{|c|c|c|c|c|c|c|c|}
\hline Patient & TS & AS & DS & QS & BS & BSSE & отU $\simeq$ \\
\hline P1 & 199924 & 188675 & 11249 & 184796 & 178145 & 120665 & 6442 \\
\hline P2 & 228803 & 189333 & 39470 & 165517 & 106264 & 106264 & 5401 है \\
\hline P3 & 190836 & 143260 & 47576 & 132649 & 127663 & 84229 & 5.087 \\
\hline P4 & 233012 & 177792 & 55220 & 175753 & 172519 & 131572 & 4831 \\
\hline P5 & 234330 & 154823 & 79507 & 151499 & 147932 & 108594 & \\
\hline Mean & 217381 & 170777 & 46604 & 162137 & 157355 & 110265 & \\
\hline
\end{tabular}

TS = total number of sequences, $\mathrm{AS}=$ number of assembled sequences, $\mathrm{DS}=$ number of discarded sequences, $\mathrm{QS}=$ quality sequences after chimera elimination, $\mathrm{BS}=$ bacterial sequences after taxonomic assignment, BSSE = bacterial sequences after singleton elimination, OTU = operational taxonomic units.

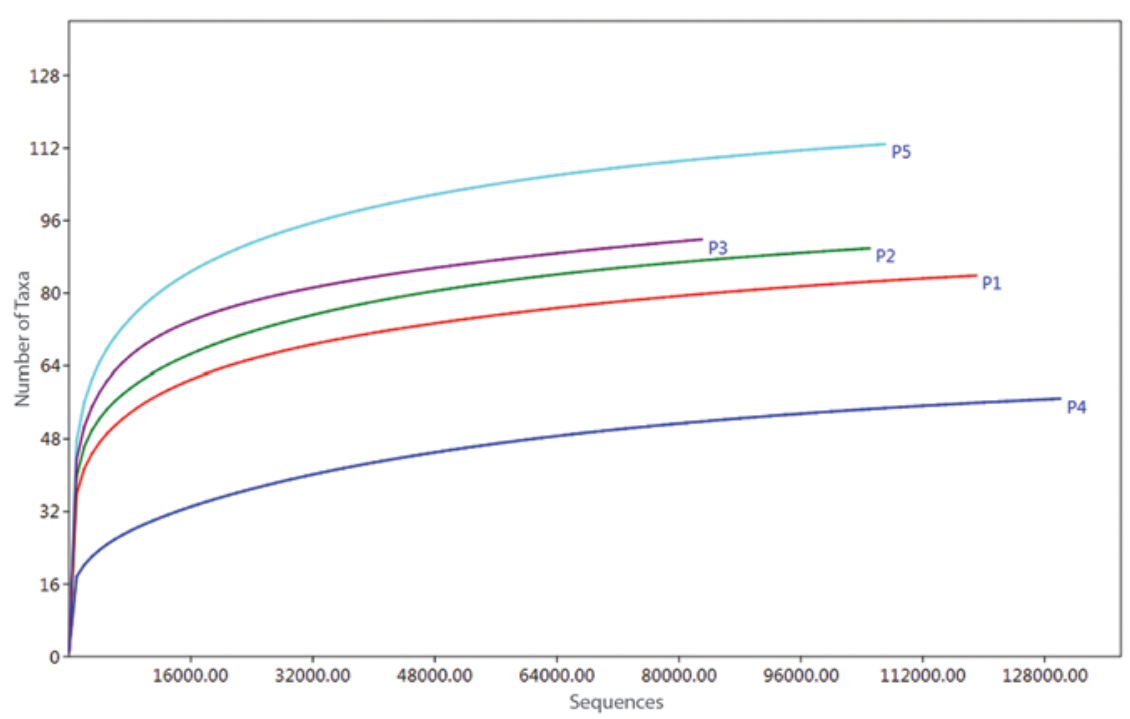

Figure 1. Depth of coverage of fecal bacterial sequences of patients with common variable immunodeficiency.

sequences from the samples was carried out with USEARCH. ${ }^{16}$ Operational taxonomic units (OTU) selection was carried out using the UCLUST ${ }^{16}$ method at $97 \%$ of similarity; a representative sequence was obtained for each OTU and taxonomy was assigned taking the EzBioCloud databases as reference. ${ }^{17}$ The OTU table was constructed in the Biological Observation Matrix (BIOM) format, ${ }^{18}$ the domains were separated and singletons were filtered (OTUs that only had one observation). ${ }^{19}$ OTU absolute abundance tables were obtained and the number of sequences was plotted by the number of taxa at the genus level, to confirm an adequate coverage depth (asymptotic trend curves); this graph was generated using PAST, version $3.15 .{ }^{20}$ The number of sequences that were simultaneously reached by all samples was 80,000 , and a simple random rarefaction process was carried out, ${ }^{21}$ taking this value as the number of sequences to be generated. This way, a standardized BIOM file was obtained for all samples. The phylum, class, order, family and genus taxonomic levels relative abundance was obtained; the phyla and genera were plotted in Excel.

\section{Results}

Total sequences mean obtained for all patients prior to assembly was 217,381 , with a mean of assembled sequences of 170,777 ; mean quality sequences after chimera removal was 162,137. After taxonomic assîfgnment, a mean of 157,355 bacterial sequences was obtained, which after singletons were eliminated:was 110,265. Mean OTU was 5679 (Table 1). Depthe of coverage in terms of the number of bacterial taxaper patient was adequate, since asymptotic tendency Ẉas achieved in each curve (Fig. 1).

Ten phyla were recorded, out of which Firmicutes ${ }^{\top}$ was the most abundant $(\bar{x}=89 \%)$, with Actinobacteria $(\bar{x}=6 \%)$ and Verrucomicrobia $(\bar{x}=3 \%)$ following in dominance (Fig. 2). Fifteen classes were determined, 


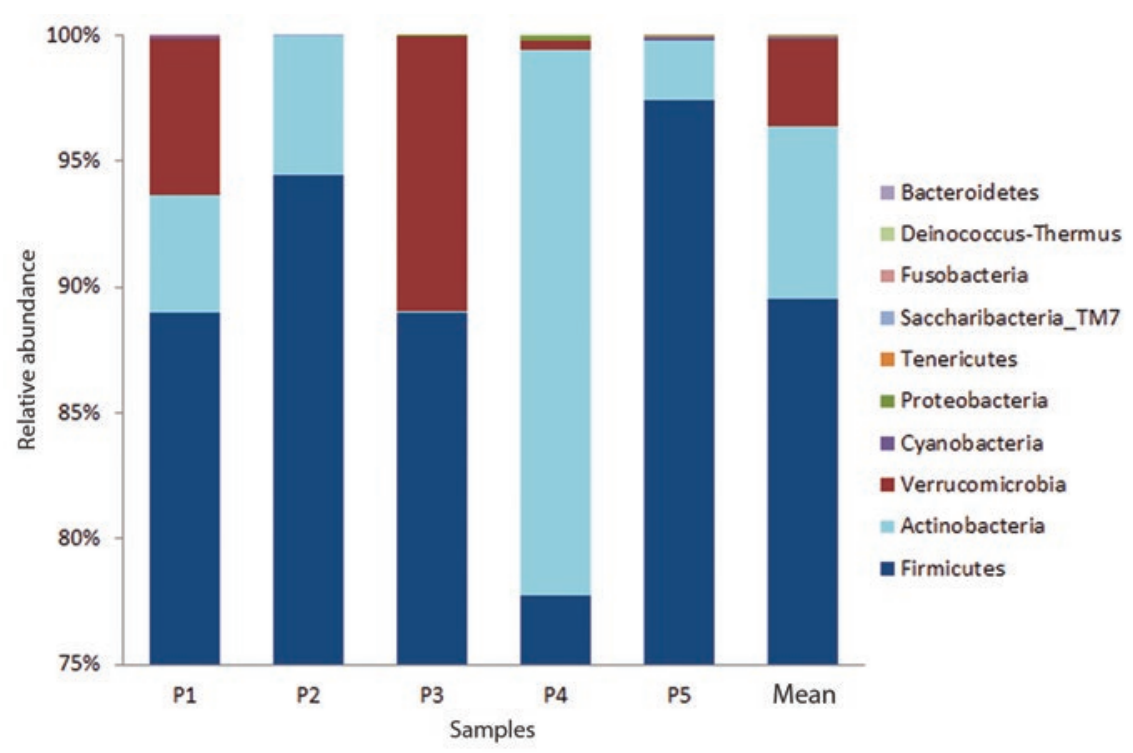

Figure 2. Relative abundance of fecal bacteria phyla of patients with common variable immunodeficiency; the last bar shows average abundaince.

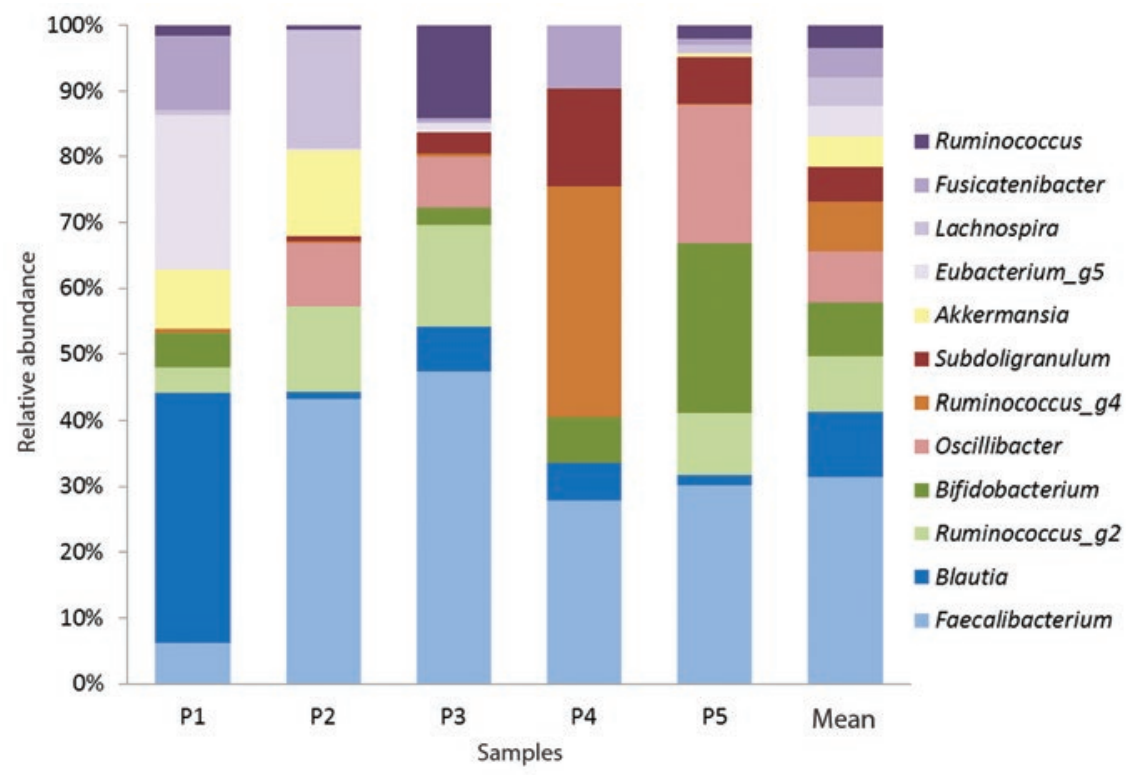

Figure 3. Relative abundance of the main fecal bacteria genera in patients with common variable immunodeficiency; the last bar shows average abundance.

with Clostridia being the most abundant ( $\bar{x}=89 \%)$, followed by an unidentified class of the Actinobacteria phylum $(\bar{x}=6 \%)$ and by the Verrucomicrobia class $(\bar{x}=3 \%)$.

Twenty-one orders were obtained, with Clostridial orders being the most abundant $(\bar{x}=89 \%)$, followed by Bifidobacterial $(\bar{x}=6 \%)$ and Verrucomicrobial $(\bar{x}=6 \%)$. Of the 34 recorded families, Ruminococcaceae $(\bar{x}=48 \%)$, Lachnospiraceae $(\bar{x}=39 \%)$, Bifidobacteriaceae $(\bar{x}=6 \%)$ and Akkermansiaceae $(\bar{x}=3 \%)$ predominated. One-hundred and sixty-six genera were reported, among which the most abundant were
Faecalibacterium $(\bar{x}=23 \%)$, Blautia $(\bar{x}=7 \%)$, Ruminococcus g2 $(\bar{x}=6.3 \%)$ and Bifidobactefium $(\bar{x}=6 \%)$ (Figure 3$)$. The identified species were Bifidobacterium longum (only species present in $100 \%$ of individuals), Bifidobacterium thermacidophilum $(20 \%$ of patients), Propionibacterium acnes (20\%), Eubacterium hallii $(20 \%)$ and Fusobacterium nucleatum $(40 \%)$

\section{Discussion}

This first approach to the study of gut microbiota in Mexican patients with CVID differs from others 
reported in healthy subjects, in whom intestinal microbiota has been reported to be mainly composed of Firmicutes and Bacteroidetes, and in less abundance of Actinobacteria, Fusobacteria, Proteobacteria, Verrucomicrobia and Cyanobacteria. 5,9,10,22,23

Firmicutes, the most abundant phylum in human gut microbiota, ${ }^{9,23}$ accounted for almost $90 \%$ of total relative abundance in the present study. Derived from this phylum, the Clostridia class, the Ruminococcaceae and Lachnospiraceae families, eight of the main bacterial genera (Faecalibacterium, Blautia, Ruminococcus, Oscillibacter, Subdoligranulum, Eubacterium, Lachnospira and Fusicatenibacter) and the Eubacterium hallii species were identified.

Clostridium IV and XIVa classes have important immune functions; their main activity is based on the production of short-chain fatty acids from dietary fiber fermentation. ${ }^{24,25}$ These short-chain fatty acids bind to G-protein-coupled receptors for free fatty acids: GPR43/FFAR2, GPRA1/FFAR3 and GPR109A, which are present in various immune cells; ${ }^{26}$ their binding stimulates interleukin-10-producing Treg CD25+ FOXP3+ and transforming growth factor beta-naïve $T$ lymphocyte differentiation. ${ }^{24,26}$ These cytokines favor the differentiation of B lymphocytes towards IgA-producing subtypes. ${ }^{25,27-29}$

The Actinobacteria phylum was the second in abundance; it includes the Actinobacteria class, the Bifidobacterial order and the Bifidobacterium genus. From this phylum, the Bifidobacterium Longum, Bifidobacterium Thermacidophilum and Propionibacterium Acnes species were identified. Bifidobacterium Longum, which was found in $100 \%$ of individuals with CVID, has probiotic activities that comprise fermenting oligosaccharides, metabolizing complex carbohydrates and participating in the biosynthesis of capsular polysaccharides that interact with Toll-2 type receptors (TLR-2) at the beginning of the innate immune response. ${ }^{29,30}$ Propinobacterium acnes ferments lactose into propionic acid, which has an antimicrobial effect. ${ }^{30-32}$ Bifidobacterium thermacidophilum has not been previously reported as part of human intestinal microbiota, however, this result should be taken with caution, since it was recorded only in one patient.

The Verrucomicrobia phylum was the third in abundance; from it, the Akkermansiaceae family and the Akkermansia genus are derived. In most studies conducted in healthy subjects (except for some in Latin American countries ${ }^{33}$ ), Verrucomicrobia is not found with high abundance; however, it was one of the most abundant in individuals with CVID. Its main immune function is to maintain the integrity of the intestinal mucus layer and produce antimicrobial peptides. $.3,34$

The low abundance of the Bacteroidetes phytum $(\bar{x}=0.00001)$ in the studied patients stands out. These gram-negative bacilli are important in the metabolism of complex polysaccharides that arrive intact to the colon. ${ }^{35}$

The Firmicutes: Bacteroidetes ratio has been proposed as an indicator of dysbiosis. ${ }^{36-38}$ It is clearthat the difference, and therefore the Firmicutes: Bacteroidetes ratio in patients with CVID of this studyare extremely high, a finding that leads to suggest intestinal dysbiosis.

In conclusion, gut microbiota composition is reported for the first time in five Mexican patients with CVID. Although most identified bacteria have a positive interaction with the immune system, the high abundance of Firmicutes and the low presence of Bacteroidetes suggest intestinal dysbiosis in these patients. Although the sample size is small, the reported findings are-expected to be the first step to delve into the study of microbiota in its relationship with the immune system. It is considered that the development of an additionnal multicenter study would be useful to reinforce-and deepen the described findings.

\section{Acknowledgements}

To the patients who participated in this study by providing the samples. To the personnel of the Laboratory of Preservation Medicine, Faculty of Biological Sciences, Universidad Juárez del Estado de Durango, Gómez Palacio, Durango, for their support in the reception, preparation and processing of samples. To the National Health Laboratory: Molecular Diagnesis and Environmental Effect on Chronic-Degenerative Diseases, Iztacala Higher Education Faculty, National Autonomous University of Mexico, Tlalnepantla, State of Mexico, for its technological and scientific support in the processing of the samples.

\section{References}

1. Bonilla FA, Barlan I, Chapel H, Costa-Carvalho BT, Cunningham-Rundles C, De la Morena MT, et al. International Consensus Doctwment (ICON): common variable immunodeficiency disorders. J Allergy Clin Immunol Pract. 2016;4:38-59.

2. Salzer U, Warnatz K, Hartmut-Peter $\mathrm{H}$. Common variable immunodeficiency. An update. Arthritis Res Ther. 2012;14:1-11.

3. Berbers R, Nierkens S, Van Laar JM Van, Bogaert D, Leavis HL. Microbial dysbiosis in common variable immune deficiencies : evidence $c a u-$ ses, and consequences. Trends Immunol. 2016;38:206-216.

4. Perreau M, Vigano S, Bellanger F, Pellaton C, Buss G, Comte D, al. Exhaustion of bacteria-specific CD4 T cells and microbial translocation in common variable immunodeficiency disorders. J Exp Med. 2014; 211:2033-2045 
5. Jandhyala SM, Talukdar R, Subramanyam C, Vuyyuru H, Sasikala M. Role of the normal gut microbiota. World J Gastroenterol. 2015;21:8787-8803.

6. Qin J, Li R, Raes J, Arumugam M, Burgdorf KS, Manichanh C, et al A human gut microbial gene catalogue established by metagenomic sequencing. Nature. 2010;464:59-65.

7. Cénit MC, Matzaraki V, Tigchelaar EF, Zhernakova A. Rapidly expanding knowledge on the role of the gut microbiome in health and disease. Biochim Biophys Acta. 2014;1842:1981-1992.

8. Belkaid Y, Hand TM. Role of the microbiota in immunity and inflamation Cell. 2014;157:121-141.

9. lizumi T, Battaglia T, Ruiz V, Pérez-Pérez Gl. Gut microbiome and antibiotics. Arch Med Res. 2017:48:727-734

10. Arumugam M, Raes J, Pelletier E, Le Paslier D, Batto J, Yamada T, et al. Enterotypes of the human gut microbiome. Nature. 2011;473:174-180.

11. Shulzhenko N, Morgun A, Hsiao W, Battle M, McCoy D, Fraser-Liggett C, et al. Crosstalk between B lymphocytes, microbiota and the intestinal ephitelium governs immunity versus metabolism in the gut. Nat Med. 2014; $17: 1585-1593$

12. Jørgensen SF, Trøseid M, Kummen M, Anmarkrud JA, Michelsen AE, Osnes LT, et al. Altered gut microbiota profile in common variable immunodeficiency associates with levels of lipopolysaccharide and markers of systemic immune activation. Mucosal Immunol. 2016:9:1455-1465.

13. Klindworth A, Pruesse E, Schweer T, Horn M, Schweer T. Evaluation of general 16 S ribosomal RNA gene PCR primers for classical and next-generation sequencing-based diversity studies. Nucleic Acids Res. 2013;41:e1

14. Caporaso JG, Kuczynski J, Stombaugh J, Bittinger K, Bushman FD, Costello EK, et al. QIIME allows analysis of high-throughput community sequencing data. Nat Methods. 2010;7:335-336.

15. Stamatakis A, Zhang J, Kobert K, Flouri T. Genome analysis PEAR : a fast and accurate Illumina Paired-End reAd mergeR. Bioinformatics. 2014;30:614-620

16. Edgar RC. Search and clustering orders of magnitude faster than BLAST. Bioinformatics. 2010;26:2460-2461

17. Yoon S, Ha S, Kwon S, Lim J, Kim Y, Seo H, et al. Introducing EzBioCloud: a taxonomically united database of 16S rRNA gene sequences and whole-genome assemblies. Int J Sysy Evol Microbiol. 2017; 1613-1617.

18. Mcdonald D, Clemente JC, Kuczynski J, Rideout JR, Stombaugh J, Wendel $D$, et al. The Biological Observation Matrix (BIOM) format or: how I learned to stop worrying and love the ome-ome. Gigascience. 2012;1(1): 7

19. Navas-Molina J, Peralta-Sánchez J, González A, McMurdie PJ, Vázquez-Baeza Y, Xu ZZ, et al. Advancing our understanding of the human microbiome using QIIME. Methods Enzymol. 2015;531:371-444.

20. Hammer $\varnothing$, Harper DAT, Ryan PD. PAST: Paleontological Statistics Software Package For Education and Data Analysis. Palaentologia Electron. 2001;4:1-9.
21. Weiss S, Xu ZZ, Peddada S, Amir A, Bittinger K, Gonzalez A, et al. Normalization and microbial differential abundance strategies depend upon data characteristics. Microbiome; 2017;5:27.

22. Ottman N, Smidt H, De Vos WM De, Belzer C. The function of otur microbiota : who is out there and what do they do? Front Cell Infect Microbiol. 2012;2:1-11.

23. Ghazalpour A, Cespedes I, Bennett BJ, Allayee H. Expanding role of gut microbiota in lipid metabolism. Curr Opin Lipidol. 2016;27:141-147

24. Lopetuso LR, Scaldaferri F, Petito V, Gasbarrini A. Commensal clostridia: leading players in the maintenance of gut homeostasis. Gut Pathog. 2013;5:1

25. Engels C, Ruscheweyh HJ, Beerenwinkel N, Lacroix C, Schwab C. The common gut microbe Eubacterium hallii also contributes to intestinal propionate formation. Front Microbiol. 2016;7:1-12.

26. Shukla SD, Budden KF, Neal R, Hansbro PM. Microbiome effects on immunity, health and disease in the lung. Clin Transl Immunology. 2017;6:133.

27. Kawamoto S, Maruya M, Kato LM, Suda W, Atarashi K, Doi Y, et al. Foxp3 + T cells regulate immunoglobulin a selection and facilitate diversification of bacterial species responsible for immune homeostasis! Immunity. 2014;41:1-14.

28. Ray A, Dittel BN. Interrelatedness between dysbiosis in the gut microbiota due to immunodeficiency and disease penetrance of colitis. Immunology. 2015;146:359-368.

29. Wu H, Wu E. Health technology slide shows. Web feature gets down to basics. Health Devices. 2003;32:126-127.

30. Ventura M, Canchaya C, Tauch A, Chandra G, Fitzgerald GF, Chater KF et al. Genomics of actinobacteria: tracing the evolutionary history of an ancient phylum. Microbiol Mol Biol Rev. 2007;71:495-548.

31. Salem I, Ramser A, Isham N, Ghannoum MA. The gut microbiomeas a major regulator of the gut-skin axis. Front Microbiol. 2018;9:1-14.

32. Achermann Y, Goldstein EJC, Coenye T, Shirtliffa ME. Propionibacterium acnes: from commensal to opportunistic biofilm-associated implant pathogen. Clin Microbiol Rev. 2014;27:419-440.

33. Fujio-Vejar S, Vasquez Y, Morales P, Magne F, Vera-Wolf P, Ugalde JA, et al. The gut microbiota of healthy Chilean subjects reveals ahigh abundance of the phylum verrucomicrobia. Front Microbiol. 2017;8:1-11.

34. Dubourg G, Lagier JC, Armougom F, Robert C, Audoly G, Papazian L, et al. High-level colonisation of the human gut by Verrucomicrobia following broad-spectrum antibiotic treatment. Int J Antimicrob Agents. 2013;41:149-155.

35. Wexler HM. Bacteroides: the good, the bad, and the nitty-gritty Clin Microbiol Rev. 2007;20:593-621.

36. Tamboli CP, Neut C, Desreumaux P, Colombel JF. Dysbiosis in ifflammatory bowel disease. Gut. 2004:53:1-4.

37. Collins SM. A role for the gut microbiota in IBS. Nat Rev Gastroenterol Hepatol. 2014;11:497-505.

38. Sampson TR, Debelius JW, Thron T, Janssen S, Shastri GG, Ihan ZE, et al. Gut microbiota regulate motor deficits and neuroinflammation in a model of Parkinson's disease. Cell. 2016;167:1469-1480. 